\title{
Strong Converse and Second-Order Asymptotics of Channel Resolvability
}

\author{
Shun Watanabe* and Masahito Hayashi ${ }^{\dagger}$ \\ *Department of Information Science and Intelligent Systems, University of Tokushima, Japan, \\ and Institute for System Research, University of Maryland, College Park. \\ Email: shun-wata@is.tokushima-u.ac.jp \\ ${ }^{\dagger}$ Graduate School of Mathematics, Nagoya University, Japan, \\ and Centre for Quantum Technologies, National University of Singapore, Singapore. \\ E-mail: masahito@math.nagoya-u.ac.jp
}

\begin{abstract}
We study the problem of channel resolvability for fixed i.i.d. input distributions and discrete memoryless channels (DMCs), and derive the strong converse theorem for any DMCs that are not necessarily full rank. We also derive the optimal second-order rate under a condition. Furthermore, under the condition that a DMC has the unique capacity achieving input distribution, we derive the optimal second-order rate of channel resolvability for the worst input distribution.
\end{abstract}

\section{INTRODUCTION}

We study the problem of channel resolvability introduced by Han-Verdú [1] (see also [2, Sec. 6.2]). In addition to theoretical interest as a random number generation problem, channel resolvability has a lot of applications in problems of information theory. First, channel resolvability can be used to show the converse coding theorem for identification via channels, and this direction of research has been extensively studied by many researchers [1], [3], [4], [5], [6]. Second, channel resolvability can be used as a building block of wiretap channel codes [7], [8], [9], [5], [10]. Third, channel resolvability can be used as a building block of channel simulation, which in turn can be used as a building block of certain coding problems (eg. [11], [12], [13], [14], [15], [16]).

Despite its importance, our understanding of channel resolvability is far from complete even for discrete memoryless channels (DMCs). For instance, the optimal rate of channel resolvability for fixed i.i.d. input distribution $p$ is not known. In [1], Han-Verdú showed it is less than or equal to the mutual information $I(p, W)$, and they also showed an example such that this bound is not tight [1, Example 1]. In [17], Han-Verdú showed that $I(p, W)$ is indeed the optimal rate for the class of channels called full rank. In this paper, we derive the optimal rate (cf. (2)) for any channels that are not necessarily full rank. In fact, we derive even stronger result, i.e., the strong converse theorem.

Once we have established the strong converse theorem, the next step is the second-order asymptotics [18], [19], [20]. In this paper, we also derive the optimal second-order rate of channel resolvability under a condition (cf. (10)). Furthermore, under the condition that a DMC has the unique capacity achieving input distribution, we derive the optimal second-order rate of channel resolvability for the worst input distribution.

The rest of this paper is organized as follows: we introduce the problem setting of channel resolvability and main results in Section II Then, we will show proofs of main results in Section [II. We conclude in Section IV and discuss open problems. The proofs of technical lemmas are given in appendices.

\section{Formulation AND Results}

\section{A. Problem Formulation}

For a given input distribution $p_{n} \in \mathcal{P}\left(\mathcal{X}^{n}\right)$ on $\mathcal{X}^{n}$ and a given channel $W: x \mapsto W_{x}$, the goal of the channel resolvability problem (for DMCs) is to approximate the output distribution

$$
W_{p_{n}}(\boldsymbol{y}):=\sum_{\boldsymbol{x} \in \mathcal{X}^{n}} p_{n}(\boldsymbol{x}) W_{\boldsymbol{x}}^{n}(\boldsymbol{y}),
$$

$W_{\boldsymbol{x}}^{n}(\boldsymbol{y})=W_{x_{1}}\left(y_{1}\right) \cdots W_{x_{n}}\left(y_{n}\right)$ is the $n$th independent extension of $W$ with input vector $\boldsymbol{x}$. Throughout the paper, we assume that alphabets are finite. More precisely, a channel resolvability code $\mathcal{C}_{n}$ of size $\left|\mathcal{C}_{n}\right|=M_{n}$ is a set of codewords $\mathcal{C}_{n}=\left\{\boldsymbol{x}_{1}, \ldots, \boldsymbol{x}_{M_{n}}\right\} \subset \mathcal{X}^{n}$, and we are interested in approximating $W_{p_{n}}$ by

$$
W_{\mathcal{C}_{n}}:=\sum_{i=1}^{M_{n}} \frac{1}{M_{n}} W_{\boldsymbol{x}_{i}}^{n}
$$

In this paper, the approximation error is evaluated by the normalized variational distance:

$$
\rho\left(\mathcal{C}_{n}, W_{p_{n}}\right):=\frac{1}{2}\left\|W_{\mathcal{C}_{n}}-W_{p_{n}}\right\|_{1} .
$$

For a given $0 \leq \varepsilon<1$, we define the minimum size of the random number needed to approximate $W_{p_{n}}$ by

$$
R\left(n, \varepsilon \mid p_{n}\right):=\inf _{\mathcal{C}_{n}}\left\{\frac{1}{n} \log \left|\mathcal{C}_{n}\right|: \rho\left(\mathcal{C}_{n}, W_{p_{n}}\right) \leq \varepsilon\right\} .
$$

We also consider the worst input distribution case:

$$
R_{\text {wst }}(n, \varepsilon):=\sup \left\{R\left(n, \varepsilon \mid p_{n}\right): p_{n} \in \mathcal{P}\left(\mathcal{X}^{n}\right)\right\},
$$

where the supremum is taken over all distributions on $\mathcal{X}^{n}$ that are not necessarily i.i.d. 


\section{B. Fixed I.I.D. Input Distribution}

First, we consider the case in which the input distribution is fixed as $p_{n}=p^{n}$ for $n$th i.i.d. extension of $p \in \mathcal{P}(\mathcal{X})$. When the transition vectors $\left\{W_{x}\right\}_{x \in \mathcal{X}}$ are linearly independent, the channel $W$ is called full rank. For full rank channels, the following result is known.

Proposition 1 ([1], [17] ): For a full rank channe 1 , we have

$$
\lim _{\varepsilon \downarrow 0} \limsup _{n \rightarrow \infty} R\left(n, \varepsilon \mid p^{n}\right)=I(p, W),
$$

where $I(p, W)$ is the mutual information for the input distribution $p$.

When a channel is not necessarily full rank, more than one $q \in \mathcal{P}(\mathcal{X})$ satisfying $W_{q}=W_{p}$ may exist. Thus, we introduce the following quantity:

$$
S_{W_{p}}:=\min \left\{I(q, W): q \in \mathcal{P}(\mathcal{X}), W_{q}=W_{p}\right\} .
$$

In general, $S_{W_{p}}$ is strictly smaller than $I(p, W)$, as is illustrated by the following example.

Example 1 ([1] $)$ : For $\mathcal{X}=\{0,1, \mathrm{e}\}$ and $\mathcal{Y}=\{0,1\}$, let $W$ be given by

$$
W_{0}(0)=1, \quad W_{1}(1)=1, \quad W_{\mathrm{e}}(0)=W_{\mathrm{e}}(1)=1 / 2 .
$$

Let $p$ be such that $p(0)=p(1)=1 / 2$. Then, we have $I(p, W)=1$ but $S_{W_{p}}=0$.

We can derive the following refinement of Proposition 1 .

Theorem 1 (First Order Asymptotics for Fixed $p$ ): For any $0<\varepsilon<1$, we have

$$
\lim _{n \rightarrow \infty} R\left(n, \varepsilon \mid p^{n}\right)=S_{W_{p}} .
$$

Fon an input distributions $q$, let

$$
U_{q, W}:=\sum_{x, y} q(x) W_{x}(y)\left[\log \frac{W_{x}(y)}{W_{q}(y)}-I(q, W)\right]^{2}
$$

and

$$
V_{q, W_{p}}:=\sum_{x, y} q(x) W_{x}(y)\left[\log \frac{W_{x}(y)}{W_{p}(y)}-D\left(W_{x} \| W_{p}\right)\right]^{2},
$$

where $D(\cdot \| \cdot)$ is the $\mathrm{KL}$ divergence. For $q$ satisfying $W_{q}=W_{p}$, $U_{q, W}$ and $V_{q, W_{p}}=V_{q, W_{q}}$ are the unconditional information variance and conditional information variance respectively [20]. In such a case, we have

$$
V_{q, W_{p}} \leq U_{q, W},
$$

and the equality hold if and only if

$$
D\left(W_{x} \| W_{p}\right)=I(q, W) \forall x \text { s.t. } q(x)>0 .
$$

Let

$$
\mathcal{V}(p, W):=\left\{q \in \mathcal{P}(\mathcal{X}): I(q, W)=S_{W_{p}}, W_{q}=W_{p}\right\} .
$$

\footnotetext{
${ }^{1}$ The full rank condition is only needed in the converse part [17].
}

Then, we define the following four quantities:

$$
\begin{aligned}
U_{p, W}^{+} & :=\max _{q \in \mathcal{V}(p, W)} U_{q, W}, \\
U_{p, W}^{-} & :=\min _{q \in \mathcal{V}(p, W)} U_{q, W}, \\
V_{p, W}^{+} & :=\max _{q \in \mathcal{V}(p, W)} V_{q, W_{p}}, \\
V_{p, W}^{-} & :=\min _{q \in \mathcal{V}(p, W)} V_{q, W_{p}} .
\end{aligned}
$$

Theorem 2 (Second Order Asymptotics for Fixed p): We have

$$
\begin{aligned}
\limsup _{n \rightarrow \infty} \sqrt{n}\left(R\left(n, \varepsilon \mid p^{n}\right)-S_{W_{p}}\right) \\
\quad \leq \begin{cases}\sqrt{U_{p, W}^{+}} Q^{-1}(\varepsilon) & \varepsilon \geq 1 / 2 \\
\sqrt{U_{p, W}^{-}} Q^{-1}(\varepsilon) & \varepsilon<1 / 2\end{cases}
\end{aligned}
$$

provided that $U_{p, W}^{-}>0$. Furthermore, if

$$
D\left(W_{x} \| W_{p}\right)=S_{W_{p}} \forall x \in \mathcal{X}
$$

and $V_{p, W}^{-}>0$ hold, we have

$$
\begin{aligned}
\lim _{n \rightarrow \infty} \sqrt{n}\left(R(n, \varepsilon)-S_{W_{p}}\right) \\
= \begin{cases}\sqrt{V_{p, W}^{+}} Q^{-1}(\varepsilon) & \varepsilon \geq 1 / 2 \\
\sqrt{V_{p, W}^{-}} Q^{-1}(\varepsilon) & \varepsilon<1 / 2\end{cases} \\
=\left\{\begin{array}{ll}
\sqrt{U_{p, W}^{+}} Q^{-1}(\varepsilon) & \varepsilon \geq 1 / 2 \\
\sqrt{U_{p, W}^{-}} Q^{-1}(\varepsilon) & \varepsilon<1 / 2
\end{array},\right.
\end{aligned}
$$

where

$$
Q(a):=\int_{a}^{\infty} \frac{1}{\sqrt{2 \pi}} \exp \left[-\frac{t^{2}}{2}\right] d t .
$$

Remark 1: In the converse part, we are going to prove the inequality $\geq$ in 111 . It should be noted that the condition in (10) is not only used as a matching condition for (11) and (12) to coincide, but it is crucially used in the converse proof. In fact, the inequality $\geq$ in 11 does not hold in general since the inequality

$$
\sqrt{V_{p, W}^{+}} Q^{-1}(\varepsilon)>\sqrt{U_{p, W}^{+}} Q^{-1}(\varepsilon)
$$

may hold for $\varepsilon>1 / 2$, which contradicts the achievability part.

Remark 2: When channel $W$ is a noiseless channel, the channel resolvability problem reduces to the source resolvability problem [21, Sec. 2]. In this case, since the channel is full rank, $\mathcal{V}(p, W)$ is the singleton $\{p\}$. We also have $S_{W_{p}}=$ $H(p), V_{p, W}^{+}=V_{p, W}^{-}=0$, and $U_{p, W}^{*}:=U_{p, W}^{+}=U_{p, W}^{-}$. Although this case is not covered by Theorem 2 the second order asymptotics for this case is already known to be [22]

$$
\limsup _{n \rightarrow \infty} \sqrt{n}\left(R\left(n, \varepsilon \mid p^{n}\right)-H(p)\right)=\sqrt{U_{p, W}^{*}} Q^{-1}(\varepsilon) .
$$




\section{Worst Input Distribution}

Next, we consider the worst input distribution case. Let

$$
C_{W}:=\max \{I(p, W): p \in \mathcal{P}(\mathcal{X})\}
$$

be the channel capacity of $W$. The following result is known.

Proposition 2 ([[] $)$ : For any $0<\varepsilon<1$, we have

$$
\lim _{n \rightarrow \infty} R_{\mathrm{wst}}(n, \varepsilon)=C_{W} \text {. }
$$

Let

$$
\mathcal{V}(W):=\left\{p \in \mathcal{P}(\mathcal{X}): I(p, W)=C_{W}\right\}
$$

be the set of all capacity achieving input distribution (CAID). It is well known that the output distribution $W_{p^{*}}$ for any CAID $p^{*}$ is unique. Let us introduce full support CAID condition:

$$
D\left(W_{x} \| W_{p^{*}}\right)=C_{W} \quad \forall x \in \mathcal{X} .
$$

Under this condition, we find that

$$
S_{W_{p^{*}}}=C_{W}
$$

holds. Moreover, $V_{p^{*}, W}^{+}$and $V_{p^{*}, W}^{-}$defined in (7) and (8) coincide with the conditional variances that appear in the channel coding problems:

$$
\begin{aligned}
V_{W}^{+} & :=\max _{p \in \mathcal{V}(W)} V_{p, W_{p}}, \\
V_{W}^{-} & :=\min _{p \in \mathcal{V}(W)} V_{p, W_{p}} .
\end{aligned}
$$

Theorem 3 (Second Order Asymptotics for the Worst Case): Suppose that the full support CAID condition is satisfied (cf. (13)). Then, we have

$$
\begin{aligned}
\limsup _{n \rightarrow \infty} \sqrt{n}\left(R_{\mathrm{wst}}(n, \varepsilon)-C_{W}\right) \\
\leq \begin{cases}\sqrt{V_{W}^{-}} Q^{-1}(\varepsilon) & \varepsilon \geq 1 / 2 \\
\sqrt{V_{W}^{+}} Q^{-1}(\varepsilon) & \varepsilon<1 / 2\end{cases}
\end{aligned}
$$

and

$$
\begin{aligned}
& \liminf _{n \rightarrow \infty} \sqrt{n}\left(R_{\mathrm{wst}}(n, \varepsilon)-C_{W}\right) \\
& \geq \begin{cases}\sqrt{V_{W}^{+}} Q^{-1}(\varepsilon) & \varepsilon \geq 1 / 2 \\
\sqrt{V_{W}^{-}} Q^{-1}(\varepsilon) & \varepsilon<1 / 2\end{cases}
\end{aligned}
$$

provided that $V_{W}^{-}>0$.

Remark 3: It should be noted that (14) is not true in general. In fact, the channel in Example 1 does not satisfy (14). It should be also noted that (14) is slightly weaker condition than (13). These conditions are needed only in the converse part, and for the achievability part of Theorem 3 , we need not to assume neither (13) nor (14).

\section{PROOFS OF MAin Results}

\section{A. Preliminaries for Proofs}

The purpose of this section is to prepare lemmas that will be used for the achievability part and the converse part, respectively. To save space, we introduce a notation that is usually used in quantum information (eg. [23]). For a function $A$ on $\mathcal{Y}$, let $\{A \geq 0\}$ indicates the set $\{y: A(y) \geq 0\}$. Then, for a non-negative function $P$ on $\mathcal{Y}$ (not necessarily normalized), we denote $P\{A \geq 0\}:=\sum_{y \in\{A \geq 0\}} P(y)$.

The following lemma guarantees existence of a good channel resolvability code.

Lemma 1 (Theorem 2 of [5]): For any $q_{n} \in \mathcal{P}\left(\mathcal{X}^{n}\right)$ such that $W_{q_{n}}=W_{p_{n}}$ and any real number $C_{n}$, there exists a channel resolvability code $\mathcal{C}_{n}$ such that

$$
\begin{aligned}
& \rho\left(\mathcal{C}_{n}, W_{p_{n}}\right) \\
& \quad \leq \sum_{\boldsymbol{x}} q_{n}(\boldsymbol{x}) W_{\boldsymbol{x}}^{n}\left\{W_{\boldsymbol{x}}^{n}-C_{n} W_{p_{n}} \geq 0\right\}+\frac{1}{2} \sqrt{\frac{C_{n}}{M_{n}}} .
\end{aligned}
$$

In the converse part, we are going to use the argument of the typical sequence. Let $T_{p, \delta}$ be the set of typical sequences, i.e., $\left|P_{\boldsymbol{x}}(a)-p(a)\right| \leq \delta \forall a \in \mathcal{X}$ and, in addition, no $a \in \mathcal{X}$ with $p(a)=0$ occur in $\boldsymbol{x}$, where $P_{\boldsymbol{x}}$ is the type of sequence $\boldsymbol{x}$. We also define the set $T_{W, \delta}(\boldsymbol{x})$ of $W$-typical sequences given $\boldsymbol{x}$, i.e., $\left|P_{\boldsymbol{x} \boldsymbol{y}}(a, b)-P_{\boldsymbol{x}}(a) W_{a}(b)\right| \leq \delta \forall(a, b) \in \mathcal{X} \times \mathcal{Y}$ and, in addition, $P_{\boldsymbol{x} \boldsymbol{y}}(a, b)=0$ whenever $W_{a}(b)=0$, where $P_{\boldsymbol{x} \boldsymbol{y}}$ is the joint type of $(\boldsymbol{x}, \boldsymbol{y})$. For the output distribution, we also define the set of typical sequences: $T_{W_{p}, \delta}$. For any $\delta>0$, it is well known that [24, Lemma 2.12]

$$
\begin{aligned}
p^{n}\left(T_{p, \delta}\right) & \geq 1-\gamma_{n}, \\
W_{p}^{n}\left(T_{W_{p}, \delta}\right) & \geq 1-\gamma_{n}, \\
W_{\boldsymbol{x}}^{n}\left(T_{W, \delta}(\boldsymbol{x})\right) & \geq 1-\gamma_{n} \forall \boldsymbol{x} \in \mathcal{X}^{n}
\end{aligned}
$$

for some $\gamma_{n}$ such that $\gamma_{n} \rightarrow 0$ as $n \rightarrow \infty$.

Let

$\mathcal{A}_{n}(\delta):=\left\{\boldsymbol{x}:\left|W_{P_{\boldsymbol{x}}}(b)-W_{p}(b)\right|>2|\mathcal{X}| \delta\right.$ for some $\left.b \in \mathcal{Y}\right\}$

be the set of all sequences such that the output distribution $W_{P_{\boldsymbol{x}}}$ is not close to $W_{p}$. For such sequences, we have the following property.

Lemma 2: For $\boldsymbol{x} \in \mathcal{A}_{n}(\delta)$, we have $T_{W, \delta}(\boldsymbol{x}) \subset T_{W_{p}, \delta^{\prime}}^{c}$ for $\delta^{\prime}=|\mathcal{X}| \delta$.

The following will be used as a key lemma in the converse part.

Lemma 3: For a given channel resolvability code $\mathcal{C}_{n}$, let $\mathcal{B}_{n}=\left\{i: \boldsymbol{x}_{i} \in \mathcal{A}_{n}(\delta)\right\}$. Then, for any $\alpha \geq 0$ and sufficiently large $n$, we have

$$
\begin{aligned}
\rho\left(\mathcal{C}_{n},\right. & \left.W_{p}^{n}\right) \\
\geq & \frac{\left|\mathcal{B}_{n}\right|}{M_{n}}\left(1-\gamma_{n}\right)+\sum_{i \in \mathcal{B}_{n}^{c}} \frac{1}{M_{n}} W_{\boldsymbol{x}_{i}}^{n}\left\{W_{\boldsymbol{x}_{i}}^{n}-e^{\alpha} M_{n} W_{p}^{n} \geq 0\right\} \\
& -e^{-\alpha}-\gamma_{n}
\end{aligned}
$$

for some $\gamma_{n}$ such that $\gamma_{n} \rightarrow 0$ as $n \rightarrow \infty$. 
The following two lemmas are also used in the converse part.

Lemma 4: Suppose $\boldsymbol{x} \notin \mathcal{A}_{n}(\delta)$. Then, we have

$$
\sum_{a} P_{\boldsymbol{x}}(a) D\left(W_{a} \| W_{p}\right)+\tau(\delta) \geq S_{W_{p}}
$$

for some $\tau(\delta)$ such that $\tau(\delta) \rightarrow 0$ as $\delta \rightarrow 0$.

Lemma 5: Suppose (10) holds and $\boldsymbol{x} \notin \mathcal{A}_{n}(\delta)$. Then, we have

$$
\begin{aligned}
& V_{P_{\boldsymbol{x}}, W_{p}}+\tau_{1}(\delta) \geq V_{p, W}^{-}, \\
& V_{P_{\boldsymbol{x}}, W_{p}}-\tau_{2}(\delta) \leq V_{p, W}^{+}
\end{aligned}
$$

for some $\tau_{1}(\delta)$ and $\tau_{2}(\delta)$ that converge to 0 as $\delta \rightarrow 0$.

\section{B. Proofs of Theorem 1$]$}

Direct Part: Let $q$ be such that $I(q, W)=S_{W_{p}}$. For arbitrarily fixed $\nu>0$, we use Lemma 1 by setting $M_{n}=$ $e^{n(I(q, W)+2 \nu)}$ and $C_{n}=e^{n(I(q, W)+\nu)}$. Then, by the law of large number, we have $\rho\left(\mathcal{C}_{n}, W_{p}^{n}\right) \rightarrow 0$. Since $\nu>0$ can be arbitrary, we complete the proof.

Converse Part: For arbitrary $0<\varepsilon<1$, suppose

$$
\liminf _{n \rightarrow \infty} R\left(n, \varepsilon \mid p^{n}\right)<S_{W_{p}} .
$$

Then, there exist $\nu>0$ and a code $\mathcal{C}_{n}$ such that $\rho\left(\mathcal{C}_{n}\right) \leq \varepsilon$ and

$$
\frac{1}{n} \log M_{n} \leq S_{W_{p}}-3 \nu
$$

for infinitely many $n$. For $q \in \mathcal{P}(\mathcal{X})$, we denote

$$
D\left(W \| W_{p} \mid q\right):=\sum_{a} q(a) D\left(W_{a} \| W_{p}\right) .
$$

From Lemma 4, if we take $\delta$ sufficiently small, we have

$$
D\left(W \| W_{p} \mid P_{\boldsymbol{x}}\right) \geq S_{W_{p}}-\nu
$$

for every $\boldsymbol{x} \notin \mathcal{A}_{n}(\delta)$.

By applying Lemma 3 for $\alpha=\nu n$, we have

$$
\begin{aligned}
\rho\left(\mathcal{C}_{n}, W_{p}^{n}\right) \\
\geq \\
\quad \frac{\left|\mathcal{B}_{n}\right|}{M_{n}}\left(1-\gamma_{n}\right)+\sum_{i \in \mathcal{B}_{n}^{c}} \frac{1}{M_{n}} W_{\boldsymbol{x}_{i}}^{n}\left\{W_{\boldsymbol{x}_{i}}^{n}-e^{\nu n} M_{n} W_{p}^{n} \geq 0\right\} \\
\quad-e^{-\nu n}-\gamma_{n} .
\end{aligned}
$$

Here, the third term and the forth term converge to 0. From (19), the second term is further lower bounded by

$$
\begin{aligned}
& \sum_{i \in \mathcal{B}_{n}^{c}} \frac{1}{M_{n}} W_{\boldsymbol{x}_{i}}^{n}\left\{\frac{1}{n} \log \frac{W_{\boldsymbol{x}_{i}}^{n}}{W_{p}^{n}} \geq S_{W_{p}}-2 \nu\right\} \\
& \quad \stackrel{\text { (a) }}{\geq} \sum_{i \in \mathcal{B}_{n}^{c}} \frac{1}{M_{n}} W_{\boldsymbol{x}_{i}}^{n}\left\{\frac{1}{n} \log \frac{W_{\boldsymbol{x}_{i}}^{n}}{W_{p}^{n}} \geq D\left(W \| W_{p} \mid P_{\boldsymbol{x}_{i}}\right)-\nu\right\},
\end{aligned}
$$

where (a) follows from 20. Here, note that

$$
\mathrm{E}_{W_{\boldsymbol{x}_{i}}^{n}}\left[\frac{1}{n} \log \frac{W_{\boldsymbol{x}_{i}}^{n}(\boldsymbol{Y})}{W_{p}^{n}(\boldsymbol{Y})}\right]=D\left(W \| W_{p} \mid P_{\boldsymbol{x}_{i}}\right)
$$

and

$$
\begin{aligned}
\mathrm{V}_{W_{\boldsymbol{x}_{i}}^{n}}\left[\frac{1}{n} \log \frac{W_{\boldsymbol{x}_{i}}^{n}(\boldsymbol{Y})}{W_{p}^{n}(\boldsymbol{Y})}\right] & =\frac{V_{P_{\boldsymbol{x}_{i}}, W_{p}}}{n} \\
& \leq \frac{\max _{q} V_{q, W_{p}}}{n}
\end{aligned}
$$

where $\mathrm{E}_{W_{\boldsymbol{x}_{i}}^{n}}$ and $\mathrm{V}_{W_{\boldsymbol{x}_{i}}^{n}}$ are the expectation and the variance with respect to $\boldsymbol{Y} \stackrel{\boldsymbol{x}_{i}}{\sim} W_{\boldsymbol{x}_{i}}^{n}$. Thus, by using Chebyshev's inequality, we have

$$
\begin{aligned}
W_{\boldsymbol{x}_{i}}^{n} & \left\{\frac{1}{n} \log \frac{W_{\boldsymbol{x}_{i}}^{n}}{W_{p}^{n}} \geq D\left(W \| W_{p} \mid P_{\boldsymbol{x}_{i}}\right)-\nu\right\} \\
\geq & 1-\frac{\max _{q} V_{q, W_{p}}}{\nu^{2} n} .
\end{aligned}
$$

Consequently, from 21, we have $\rho\left(\mathcal{C}_{n}, W_{p}^{n}\right) \rightarrow 1$, which contradict with $\rho\left(\mathcal{C}_{n}, W_{p}^{n}\right) \leq \varepsilon$. Thus, we have $\liminf _{n \rightarrow \infty} R(n, \varepsilon) \geq S_{W_{p}}$.

\section{Proofs of Theorem 2}

Direct Part: Let $q$ be such that $I(q, W)=S_{W_{p}}$ and $U_{q, W}=U_{q, W}^{-}$(or $\left.U_{q, W}=U_{q, W}^{+}\right)$. For arbitrarily fixed $\nu>0$, we use Lemma 1 by setting $\log M_{n}=n I(q, W)+$ $\sqrt{n U_{q, W}} Q^{-1}(\varepsilon-\nu)+\log n$ and $\log C_{n}=n I(q, W)+$ $\sqrt{n U_{q, W}} Q^{-1}(\varepsilon-\nu)$. Then, by the central limit theorem, we have $\rho\left(\mathcal{C}_{n}, W_{p}^{n}\right) \leq \varepsilon$ for sufficiently large $n$. Since $\nu>0$ can be arbitrary, we complete the proof of 9 .

Converse Part: We only prove' the case with $\varepsilon<1 / 2$. Suppose

$$
\liminf _{n \rightarrow \infty} \sqrt{n}\left(R\left(n, \varepsilon \mid p^{n}\right)-n S_{W_{p}}\right)<\sqrt{V_{p, W}^{-}} Q^{-1}(\varepsilon) .
$$

Then, there exists $\nu>0$ and a code $\mathcal{C}_{n}$ such that $\rho\left(\mathcal{C}_{n}\right) \leq \varepsilon$ and

$$
\log M_{n} \leq n S_{W_{p}}+\sqrt{n V_{p, W}^{-}} Q^{-1}(\varepsilon)-3 \nu \sqrt{n}
$$

for infinitely many $n$. From (17) of Lemma 5 if we take $\delta$ sufficiently small, we have

$$
\sqrt{V_{P_{\boldsymbol{x}}, W_{p}}} Q^{-1}(\varepsilon) \geq \sqrt{V_{p, W}^{-}} Q^{-1}(\varepsilon)-\nu
$$

for ever $\boldsymbol{x} \notin \mathcal{A}_{n}(\delta)$.

By applying Lemma 3 for $\alpha=\nu \sqrt{n}$, we have

$$
\begin{aligned}
& \rho\left(\mathcal{C}_{n}, W_{p}^{n}\right) \geq \\
& \quad \frac{\left|\mathcal{B}_{n}\right|}{M_{n}}\left(1-\gamma_{n}\right)+\sum_{i \in \mathcal{B}_{n}^{c}} \frac{1}{M_{n}} W_{\boldsymbol{x}_{i}}^{n}\left\{W_{\boldsymbol{x}_{i}}^{n}-e^{\nu \sqrt{n}} M_{n} W_{p}^{n} \geq 0\right\} \\
& \quad-e^{-\nu \sqrt{n}}-\gamma_{n} .
\end{aligned}
$$

${ }^{2}$ For $\varepsilon>1 / 2$, we replace $V_{p, W}^{-}$in 26 by $V_{p, W}^{+}$, which follows from 18 of Lemma 5 by noting $Q^{-1}(\varepsilon)<0$ for $\varepsilon>1 / 2$. 
From [25, each term in the summation of the second term is further lower bounded by

$W_{\boldsymbol{x}_{i}}^{n}\left\{\frac{1}{\sqrt{n}}\left(\log \frac{W_{\boldsymbol{x}_{i}}^{n}}{W_{p}^{n}}-n S_{W_{p}}\right) \geq \sqrt{V_{p, W}^{-}} Q^{-1}(\varepsilon)-2 \nu\right\}$

(a)

$W_{\boldsymbol{x}_{i}}^{n}\left\{\frac{1}{\sqrt{n}}\left(\log \frac{W_{\boldsymbol{x}_{i}}^{n}}{W_{p}^{n}}-n S_{W_{p}}\right) \geq \sqrt{V_{P_{\boldsymbol{x}_{i}}, W_{p}}} Q^{-1}(\varepsilon)-\nu\right\}$,

where (a) follows from (26). Here, we note that $D\left(W \| W_{p} \mid P_{\boldsymbol{x}}\right)=S_{W_{p}}$ holds for any sequence $\boldsymbol{x}$ because of the assumption in (10). Now, by noting (22) and 23, and by using the central limit theorem, (28) is strictly larger than $\varepsilon$ for sufficiently large $n$. Thus, from 27), we have $\rho\left(\mathcal{C}_{n}, W_{p}^{n}\right)>\varepsilon$ for sufficiently large $n$, which is a contradiction. Thus, we have

$$
\liminf _{n \rightarrow \infty} \sqrt{n}\left(R\left(n, \varepsilon \mid p^{n}\right)-n S_{W_{p}}\right) \geq \sqrt{V_{p, W}^{-}} Q^{-1}(\varepsilon),
$$

which completes the proof of $\geq$ in (11). The equality between (11) and (12) follows from the assumption in (10).

\section{Proof of Theorem 3}

Direct Part: Let $p^{*}$ be CAID, and let $V_{W}=V_{W}^{+}$when $\varepsilon<1 / 2$ (or $V_{W}^{-}$when $\varepsilon \geq 1 / 2$ ). From Lemma1 with $q_{n}=p_{n}$, there exists a resolvability code satisfying

$$
\begin{aligned}
& \rho\left(\mathcal{C}_{n}, W_{p_{n}}\right) \\
& \quad \leq \sum_{\boldsymbol{x}} p_{n}(\boldsymbol{x}) W_{\boldsymbol{x}}^{n}\left\{\log \frac{W_{\boldsymbol{x}}^{n}}{W_{p_{n}}} \geq \log C_{n}\right\}+\frac{1}{2} \sqrt{\frac{C_{n}}{M_{n}}} .
\end{aligned}
$$

Here, by the change of measure argument, we have

$$
\begin{aligned}
& W_{\boldsymbol{x}}^{n}\left\{\log \frac{W_{\boldsymbol{x}}^{n}}{W_{p_{n}}} \geq \log C_{n}\right\} \\
& =W_{\boldsymbol{x}}^{n}\left\{\log \frac{W_{\boldsymbol{x}}^{n}}{W_{p^{*}}^{n}}+\log \frac{W_{p^{*}}^{n}}{W_{p_{n}}} \geq \log C_{n}\right\} \\
& \leq W_{\boldsymbol{x}}^{n}\left\{\log \frac{W_{\boldsymbol{x}}^{n}}{W_{p^{*}}^{n}} \geq \log C_{n}-\xi\right\}+W_{\boldsymbol{x}}^{n}\left\{\log \frac{W_{p^{*}}^{n}}{W_{p_{n}}} \geq \xi\right\}
\end{aligned}
$$

for any $\xi>0$, which implies

$$
\begin{aligned}
& \sum_{\boldsymbol{x}} p_{n}(\boldsymbol{x}) W_{\boldsymbol{x}}^{n}\left\{\log \frac{W_{\boldsymbol{x}}^{n}}{W_{p_{n}}} \geq \log C_{n}\right\} \\
& \leq \sum_{\boldsymbol{x}} p_{n}(\boldsymbol{x}) W_{\boldsymbol{x}}^{n}\left\{\log \frac{W_{\boldsymbol{x}}^{n}}{W_{p^{*}}^{n}} \geq \log C_{n}-\xi\right\}+e^{-\xi} .
\end{aligned}
$$

Now, for arbitrarily fixed $\nu>0$, let $\xi=\log n, \log M_{n}=$ $n C_{W}+\sqrt{n V_{W}} Q^{-1}(\varepsilon-\nu)+2 \log n$ and $\log C_{n}=n C_{W}+$ $\sqrt{n V_{W}} Q^{-1}(\varepsilon-\nu)+\log n$. Then, by applying the central limit theorem for each $W_{\boldsymbol{x}}^{n}\{\cdot\}$ in $(29)$, we have $\rho\left(\mathcal{C}_{n}, W_{p_{n}}\right) \leq \varepsilon$ for sufficiently large $n$. Since $\nu>0$ can be arbitrary, we complete the proof of the direct part. have

Converse Part: From the definition of the worst case, we

$$
R_{\mathrm{wst}}(n, \varepsilon) \geq R\left(n, \varepsilon \mid\left(p^{*}\right)^{n}\right) .
$$

Thus, the converse part follows from Theorem 2 .

\section{CONCLUSION}

As we discussed in Remark 1, the optimal second-order rate for fixed i.i.d. input distribution is not clear in general. One possible answer is that the optimal second-order rate is always given by (12). This is at least true for noiseless channel (cf. Remark 2), but there is no strong evidence in general. Clarifying the optimal second-order rate is an important future research agenda. There is also a gap between the achievability and the converse for the worst input distribution case in general (cf. Theorem 3); the gap vanishes only when the channel has the unique CAID.

\section{APPENDIX}

\section{A. Proof of Lemma 2}

From the definition of $T_{W, \delta}(\boldsymbol{x}), \boldsymbol{y} \in T_{W, \delta}(\boldsymbol{x})$ implies $\left|P_{\boldsymbol{y}}(b)-W_{P_{\boldsymbol{x}}}(b)\right| \leq \delta^{\prime} \forall b \in \mathcal{Y}$. On the other hand, from the definition of $\mathcal{A}_{n}(\delta)$, there exists $b \in \mathcal{Y}$ such that

$$
\left|W_{P_{\boldsymbol{x}}}(b)-W_{p}(b)\right|>2 \delta^{\prime} .
$$

Thus, for $b$ satisfying $\underline{30}, \boldsymbol{y} \in T_{W, \delta}(\boldsymbol{x})$ implies

$$
\begin{aligned}
& \left|P_{\boldsymbol{y}}(b)-W_{p}(b)\right| \\
& \quad \geq\left|W_{P_{\boldsymbol{x}}}(b)-W_{p}(b)\right|-\left|P_{\boldsymbol{y}}(b)-W_{P_{\boldsymbol{x}}}(b)\right| \\
& \quad>\delta^{\prime},
\end{aligned}
$$

which implies $\boldsymbol{y} \notin T_{W_{p}, \delta^{\prime}}$.

\section{B. Proof of Lemma 3}

First, we divide $W_{p}^{n}$ into typical part and non-typical part as $W_{p}^{n}=\hat{W}_{p}^{n}+\tilde{W}_{p}^{n}$, where

$$
\begin{aligned}
& \hat{W}_{p}^{n}(\boldsymbol{y}):=W_{p}^{n}(\boldsymbol{y}) \mathbf{1}\left[\boldsymbol{y} \in T_{W_{p}, \delta^{\prime}}\right], \\
& \tilde{W}_{p}^{n}(\boldsymbol{y}):=W_{p}^{n}(\boldsymbol{y}) \mathbf{1}\left[\boldsymbol{y} \notin T_{W_{p}, \delta^{\prime}}\right],
\end{aligned}
$$

where $\delta^{\prime}$ is specified in Lemma 2 and $\mathbf{1}[\cdot]$ is the indicator function. Then, for sufficiently large $n$, we have

$$
\begin{aligned}
& \frac{1}{2}\left\|W_{\mathcal{C}_{n}}-W_{p}^{n}\right\|_{1} \\
& \quad \stackrel{\text { (a) }}{\geq} W_{\mathcal{C}_{n}}\left\{W_{\mathcal{C}_{n}}-e^{\alpha} \hat{W}_{p}^{n} \geq 0\right\}-W_{p}^{n}\left\{W_{\mathcal{C}_{n}}-e^{\alpha} \hat{W}_{p}^{n} \geq 0\right\} \\
& \quad \geq \quad W_{\mathcal{C}_{n}}\left\{W_{\mathcal{C}_{n}}-e^{\alpha} \hat{W}_{p}^{n} \geq 0\right\}-\hat{W}_{p}^{n}\left\{W_{\mathcal{C}_{n}}-e^{\alpha} \hat{W}_{p}^{n} \geq 0\right\} \\
& \quad-\tilde{W}_{p}^{n}\left(\mathcal{Y}^{n}\right)
\end{aligned}
$$

where (a) follows form the definition of the variational distance, and (b) follows from

$$
\begin{aligned}
\hat{W}_{p}^{n}\left\{W_{\mathcal{C}_{n}}-e^{\alpha} \hat{W}_{p}^{n} \geq 0\right\} & \leq e^{-\alpha} W_{\mathcal{C}_{n}}\left\{W_{\mathcal{C}_{n}}-e^{\alpha} \hat{W}_{p}^{n} \geq 0\right\} \\
& \leq e^{-\alpha}
\end{aligned}
$$

and $\tilde{W}_{p}^{n}\left(\mathcal{Y}^{n}\right)=W_{p}^{n}\left(T_{W_{p}, \delta^{\prime}}^{c}\right) \leq \gamma_{n}$ for sufficiently large $n$. 
Furthermore, we have

$$
\begin{aligned}
& W_{\mathcal{C}_{n}}\left\{W_{\mathcal{C}_{n}}-e^{\alpha} \hat{W}_{p}^{n} \geq 0\right\} \sum_{i=1}^{M_{n}} \frac{1}{M_{n}} W_{\boldsymbol{x}_{i}}^{n}\left\{\sum_{j=1}^{M_{n}} W_{\boldsymbol{x}_{j}}^{n}-e^{\alpha} M_{n} \hat{W}_{p}^{n} \geq 0\right\} \\
& \stackrel{(\mathrm{c})}{\geq} \sum_{i=1}^{M_{n}} \frac{1}{M_{n}} W_{\boldsymbol{x}_{i}}^{n}\left\{W_{\boldsymbol{x}_{i}}^{n}-e^{\alpha} M_{n} \hat{W}_{p}^{n} \geq 0\right\} \\
&=\sum_{i \in \mathcal{B}_{n}} \frac{1}{M_{n}} W_{\boldsymbol{x}_{i}}^{n}\left\{W_{\boldsymbol{x}_{i}}^{n}-e^{\alpha} M_{n} \hat{W}_{p}^{n} \geq 0\right\} \\
& \quad+\sum_{i \in \mathcal{B}_{n}^{c}} \frac{1}{M_{n}} W_{\boldsymbol{x}_{i}}^{n}\left\{W_{\boldsymbol{x}_{i}}^{n}-e^{\alpha} M_{n} \hat{W}_{p}^{n} \geq 0\right\},
\end{aligned}
$$

where (c) follows from the fact that

$$
\left\{W_{\boldsymbol{x}_{i}}^{n}-e^{\alpha} M_{n} \hat{W}_{p}^{n} \geq 0\right\} \subset\left\{\sum_{j=1}^{M_{n}} W_{\boldsymbol{x}_{j}}^{n}-e^{\alpha} M_{n} \hat{W}_{p}^{n} \geq 0\right\}
$$

holds for each $i$.

Now, we evaluate each term of (32) separately. Since $\boldsymbol{x}_{i} \in$ $\mathcal{A}_{n}(\delta)$ for $i \in \mathcal{B}_{n}$, from Lemma 2, we have $\hat{W}_{p}^{n}(\boldsymbol{y})=0$ for $\boldsymbol{y} \in T_{W, \delta}\left(\boldsymbol{x}_{i}\right)$, which implies

$$
T_{W, \delta}\left(\boldsymbol{x}_{i}\right) \subset\left\{W_{\boldsymbol{x}_{i}}^{n}-e^{\alpha} M_{n} \hat{W}_{p}^{n} \geq 0\right\} .
$$

Thus, the first term is lower bounded as

$$
\begin{aligned}
& \sum_{i \in \mathcal{B}_{n}} \frac{1}{M_{n}} W_{\boldsymbol{x}_{i}}^{n}\left\{W_{\boldsymbol{x}_{i}}^{n}-e^{\alpha} M_{n} \hat{W}_{p}^{n} \geq 0\right\} \\
& \geq \sum_{i \in \mathcal{B}_{n}} \frac{1}{M_{n}} W_{\boldsymbol{x}_{i}}^{n}\left(T_{W, \delta}\left(\boldsymbol{x}_{i}\right)\right) \\
& \geq \frac{\left|\mathcal{B}_{n}\right|}{M_{n}}\left(1-\gamma_{n}\right)
\end{aligned}
$$

for sufficiently large $n$. On the other hand, since

$$
\left\{W_{\boldsymbol{x}_{i}}^{n}-e^{\alpha} M_{n} W_{p}^{n} \geq 0\right\} \subset\left\{W_{\boldsymbol{x}_{i}}^{n}-e^{\alpha} M_{n} \hat{W}_{p}^{n} \geq 0\right\},
$$

the second term is lower bounded as

$$
\begin{aligned}
& \sum_{i \in \mathcal{B}_{n}^{c}} \frac{1}{M_{n}} W_{\boldsymbol{x}_{i}}^{n}\left\{W_{\boldsymbol{x}_{i}}^{n}-e^{\alpha} M_{n} \hat{W}_{p}^{n} \geq 0\right\} \\
& \quad \geq \sum_{i \in \mathcal{B}_{n}^{c}} \frac{1}{M_{n}} W_{\boldsymbol{x}_{i}}^{n}\left\{W_{\boldsymbol{x}_{i}}^{n}-e^{\alpha} M_{n} W_{p}^{n} \geq 0\right\} .
\end{aligned}
$$

Finally, by combining (31)- 34), we have the desired bound.

\section{Proof of Lemma 4}

Let

$$
\mathcal{Q}(\delta):=\left\{q:\left|W_{q}(b)-W_{p}(b)\right| \leq 2|\mathcal{X}| \delta \forall b \in \mathcal{Y}\right\} .
$$

Then, from the definition of $\mathcal{A}_{n}(\delta)$, we have

$$
\sum_{a} P_{\boldsymbol{x}}(a) D\left(W_{a} \| W_{p}\right) \geq \min _{q \in \mathcal{Q}(\delta)} \sum_{a} q(a) D\left(W_{a} \| W_{p}\right) .
$$

Since the righthand side of (36) is a linear programming, by the perturbation analysis [25, Sec. 5.6.2], we have

$$
\begin{aligned}
\min _{q \in \mathcal{Q}(\delta)} & \sum_{a} q(a) D\left(W_{a} \| W_{p}\right) \\
& \geq \min _{q \in \mathcal{Q}(0)} \sum_{a} q(a) D\left(W_{a} \| W_{p}\right)-\tau(\delta) \\
& =S_{W_{p}}-\tau(\delta)
\end{aligned}
$$

for some $\tau(\delta)$ such that $\tau(\delta) \rightarrow 0$ as $\delta \rightarrow 0$.

\section{Proof of Lemma 5}

Since (10) holds, we have $\mathcal{V}(p, W)=\mathcal{Q}(0)$, where $\mathcal{Q}(\delta)$ is defined by (35). Thus, we have

$$
V_{p, W}^{-}=\min _{q \in \mathcal{Q}(0)} V_{q, W_{p}}, \quad V_{p, W}^{+}=\max _{q \in \mathcal{Q}(0)} V_{q, W_{p}} .
$$

We also have

$$
\begin{aligned}
& V_{P_{\boldsymbol{x}}, W_{p}} \geq \min _{q \in \mathcal{Q}(\delta)} V_{q, W_{p}}, \\
& V_{P_{\boldsymbol{x}}, W_{p}} \leq \max _{q \in \mathcal{Q}(\delta)} V_{q, W_{p}}
\end{aligned}
$$

for $\boldsymbol{x} \notin \mathcal{A}_{n}(\delta)$. Since the righthand sides of (37) and 38) are linear programmings, we can show the statement of the lemma in the same reason as Lemma 4.

\section{REFERENCES}

[1] T. S. Han and S. Verdú, "Approximation theory of output statistics," IEEE Trans. Inform. Theory, vol. 39, no. 3, pp. 752-772, May 1993.

[2] A. D. Wyner, "The common information of two dependent random variables," IEEE Trans. Inform. Theory, vol. 21, no. 2, pp. 163-179, March 1975.

[3] Y. Steinberg, "New converses in the theory of identification via channels," IEEE Trans. Inform. Theory, vol. 44, no. 3, pp. 984-998, May 1998.

[4] R. Ahlswede and A. Winter, "Strong converse for identification via quantum channels," IEEE Trans. Inform. Theory, vol. 48, no. 3, pp. 569-579, March 2002.

[5] M. Hayashi, "General nonasymptotic and asymptotic formulas in channel resolvability and identification capacity and their application to the wiretap channel," IEEE Trans. Inform. Theory, vol. 52, no. 4, pp. 1562 1575, April 2006.

[6] Y. Oohama, "Converse coding theorems for identification via channels," IEEE Trans. Inform. Theory, vol. 59, no. 2, pp. 744-759, February 2013.

[7] I. Csiszár, "Almost independence and secrecy capacity," Problems of Information Transmission, vol. 32, no. 1, pp. 40-47, 1996.

[8] N. Cai, A. Winter, and R. W. Yeung, "Quantum privacy and quantum wiretap channels," Problems of Information Transmission, vol. 40, no. 4, pp. $26-47,2004$.

[9] I. Devetak, "The private classical capacity and quantum capacity of a quantum channel," IEEE Trans. Inform. Theory, vol. 51, no. 1, pp. 4455, January 2005, arXiv:quant-ph/0304127.

[10] M. Bloch and J. N. Laneman, "Strong secrecy from channel resolvability," IEEE Trans. Inform. Theory, vol. 59, no. 12, pp. 8077-8098, December 2013.

[11] C. H. Bennett, P. W. Shor, J. A. Smolin, and A. V. Thapliyal, "Entanglement-assisted capacity of a quantum channel and the reverse Shannon theorem," IEEE Trans. Inform. Theory, vol. 48, no. 10, pp. 2637-2655, October 2002.

[12] A. Winter, "Compression of sources of probability distributions and density operators," 2002, arXiv:quant-ph/0208131.

[13] Z. Luo and I. Devetak, "Channel simulation with quantum side information," IEEE Trans. Inform. Theory, vol. 55, no. 3, pp. 1331-1342, March 2009.

[14] N. Datta, M. H. Hsieh, and M. M. Wilde, "Quantum rate distortion, reverse Shannon theorem, and source-channel separation," IEEE Trans. Inform. Theory, vol. 59, no. 1, pp. 615-630, January 2013. 
[15] P. Cuff, "Distributed channel synthesis," IEEE Trans. Inform. Theory, vol. 59, no. 11, pp. 7071-7096, November 2013.

[16] S. Watanabe, S. Kuzuoka, and V. Y. F. Tan, "Non-asymptotic and secondorder achievability bounds for coding with side-informationn," 2013, arXiv:1301.6467.

[17] T. S. Han and S. Verdú, "Spectrum invariancy under output approximation for full-rank discrete memoryless channels," Problemy Peredachi Informatsii, vol. 29, no. 2, pp. 9-27, 1993.

[18] V. Strassen, "Asymptotische Abschätzungen in Shannons Informationstheorie," in Trans. Third. Prague Conf. Inf. Th., 1962, pp. 689-723.

[19] M. Hayashi, "Information spectrum approach to second-order coding rate in channel coding," IEEE Trans. Inform. Theory, vol. 55, no. 11, pp. 4947-4966, November 2009.

[20] Y. Polyanskiy, H. V. Poor, and S. Verdu, "Channel coding rate in the finite blocklength regime," IEEE Trans. Inform. Theory, vol. 56, no. 5, pp. 2307-2359, May 2010.

[21] T. S. Han, Information-Spectrum Methods in Information Theory. Springer, 2003.

[22] R. Nomura and T. S. Han, "Second-order resolvability, intrinsic randomness, and fixed-length source coding for mixed sources: Information spectrum approach," IEEE Trans. Inform. Theory, vol. 59, no. 1, pp. 116, January 2013.

[23] H. Nagaoka and M. Hayashi, "An information-spectrum approach to classical and quantum hypothesis testing for simple hypotheses," IEEE Trans. Inform. Theory, vol. 53, no. 2, pp. 534-549, February 2007.

[24] I. Csiszár and J. Körner, Information Theory, Coding Theorems for Discrete Memoryless Systems, 2nd ed. Cambridge University Press, 2011.

[25] S. Boyd and L. Vandenberghe, Convex Optimization. Cambridge University Press, 2004. 